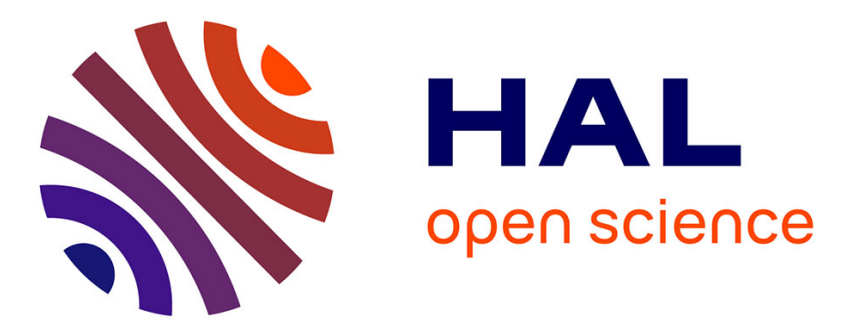

\title{
Thermionic cooling devices based on resonant-tunneling AlGaAs/GaAs heterostructure
}

\author{
M. Bescond, D. Logoteta, M Michelini, N. Cavassilas, T Yan, A. Yangui, M. \\ Lannoo, K. Hirakawa
}

\section{- To cite this version:}

M. Bescond, D. Logoteta, M Michelini, N. Cavassilas, T Yan, et al.. Thermionic cooling devices based on resonant-tunneling AlGaAs/GaAs heterostructure. Journal of Physics: Condensed Matter, 2018, 30 (6), 10.1088/1361-648X/aaa4cf . hal-01761821

\section{HAL Id: hal-01761821 \\ https://hal.science/hal-01761821}

Submitted on 25 Jan 2022

HAL is a multi-disciplinary open access archive for the deposit and dissemination of scientific research documents, whether they are published or not. The documents may come from teaching and research institutions in France or abroad, or from public or private research centers.
L'archive ouverte pluridisciplinaire $\mathbf{H A L}$, est destinée au dépôt et à la diffusion de documents scientifiques de niveau recherche, publiés ou non, émanant des établissements d'enseignement et de recherche français ou étrangers, des laboratoires publics ou privés.

\section{(이) $\$$}

Distributed under a Creative Commons Attribution - NonCommercial - NoDerivatives| 4.0 


\title{
Thermionic cooling devices based on resonant-tunneling AlGaAs/GaAs heterostructure
}

\author{
M. Bescond,${ }^{1, *}$ D. Logoteta, ${ }^{2}$ F. Michelini, ${ }^{2}$ N. Cavassilas, ${ }^{2}$ \\ T. Yan, ${ }^{1}$ A. Yangui, ${ }^{1}$ M. Lannoo, ${ }^{2}$ and K. Hirakawa ${ }^{1}$ \\ ${ }^{1}$ LIMMS, CNRS-Institute of Industrial Science, UMI \\ 2820, University of Tokyo, 153-8505 Tokyo, Japan \\ ${ }^{2}$ IM2NP, UMR CNRS 7334, Aix-Marseille Université, Technopôle de Château-Gombert, \\ Bâtiment Néel, 60 Rue Frédéric Joliot Curie, 13453 Marseille, France
}

\begin{abstract}
We study by means of full quantum simulations the operating principle and performance of a semiconductor heterostructure refrigerator combining resonant tunneling filtering and thermionic emission. Our model takes into account the coupling between the electric and thermal currents by self-consistently solving the transport equations within the non-equilibrium Green's function framework and the heat equation. We show that the device can achieve relatively high cooling power values, while, in the considered implementation, the maximum lattice temperature drop is severely limited by the thermal conductivity of the constituting materials. In such out-ofequilibrium structure, we then emphasize the significant deviation of the phonon temperature from its electronic counterpart which can vary over several hundred Kelvin. The interplay between those two temperatures and the impact on the electrochemical potential is also discussed. Finally, viable options toward an optimization of the device are proposed.
\end{abstract}




\section{INTRODUCTION}

Handling the rapid increase of the heat power densities associated to the electronic miniaturization is a major scientific and industrial issue. In microelectronics, self-heating effects result, indeed, in significant reductions of both transistor efficiency ${ }^{1,2}$ and lifetime ${ }^{3}$. Similar difficulties arise also in the case of optoelectronic devices, particularly those operating with intense radiation, as light-concentrating solar cells ${ }^{4}$. The development of embedded systems in a Internet of Things perspective will, moreover, require local, and possibly on-chip, thermal management capabilities ${ }^{5-7}$.

Temperature stabilization by means of electronic refrigerators has attracted much interest, as a scalable, reliable and "green" option. The researches in this field have mainly focused on two approaches, which rely either on thermoelectricity or thermionic emission of hot carriers.

Thermoelectric devices are based on the Peltier effect. Their performance can be quantified through the dimensionless figure of merit $Z T=\sigma S^{2} T /\left(\kappa_{e}+\kappa_{l}\right)$, where $\sigma$ is the electronic conductivity, $S$ is the Seebeck coefficient, $T$ is the average temperature of the hot and cold side of the device, and $\kappa_{e}$ and $\kappa_{l}$ are the electronic and lattice thermal conductivities, respectively. An efficient thermoelectric system must therefore have a high $\sigma$ (to minimize the Joule effect), low $\kappa_{e}$ and $\kappa_{l}$ (to avoid thermal short-circuit), and a high $S$ (to maximize the conversion of electrical energy to temperature variation, or vice versa). Despite extensive research efforts, for over fifty years the best thermoelectric materials had $Z T \approx 1$. In 1993 , the pioneering work of Hicks and Dresselhaus ${ }^{8}$ paved the way to the exploitation of nanoscale effects to improve the thermoelectric device efficiency. Since then, experimental studies, e.g. on thin-film superlattices ${ }^{9}$, quantum-dot superlattices ${ }^{10}$ and nanowires ${ }^{11,12}$, reported higher values of $Z T$, mostly due to the substantial reduction of the phonon thermal conductivity in nanostructures ${ }^{13-15}$.

Thermionic devices were proposed as an alternative to thermoelectric devices, able to reduce the effects of carrier scattering and lattice thermal conduction. The operation of these devices is based on the extraction of thermally excited electrons from an emitter (the cold side of the device), via thermionic emission over a potential barrier. The carriers, then, almost ballistically reach the collector (the hot side), where they thermalize at lower 
energies. A voltage generator sustains a current in the device, by supplying cold carriers from the collector to the emitter. It has been pointed out that the working principle of thermionic devices can be described as well in terms of Peltier cooling and heating at the emitter-barrier and barrier-collector interfaces, respectively, and that the physical quantities defining their performance are the same as those entering the expression of $Z T^{16}$. Nevertheless, the performance of thermionic devices is traditionally assessed by referring to the more device-oriented Coefficient Of Performance $\mathrm{COP}=J_{Q} /(J V)$, where $J_{Q}$ is the heat power density per unit area extracted from the cold side, and $J V$ is the the density of power supplied to the device ( $J$ is the electrical current density and $V$ the voltage bias).

Within the same miniaturization trend as for their thermoelectric counterparts, solid state implementation of these devices based on heterostructures have been envisaged. The first solid state thermionic refrigerator (analogous of a vacuum diode) has been proposed by Mahan ${ }^{17-19}$. Currently, other options are being investigated, as it has been pointed out that simple single-barrier thermionic devices cannot exhibit performance higher than their bulk thermoelectric counterparts ${ }^{16,20,21}$. In particular, on the basis of the theoretical predictions in Refs.22-29, the energy filtering properties of multibarrier structures (typically in the form of superlattices) have been successfully exploited. With respect to thermoelectric coolers, these devices are expected to offer advantages in terms of compatibility with standard integration processes, high cooling power density and fast transient response ${ }^{30}$.

In this paper, we study a AlGaAs/GaAs heterostructure-based thermionic refrigerator first proposed by Chao et al. ${ }^{31}$, which exploits resonant tunneling filtering to enhance the cooling at the interface with a thermionic barrier. Our numerical full quantum simulations are able to take into account the thermal effects by self-consistently coupling the electron transport equations within the non-equilibrium Green's function (NEGF) formalism with the heat equation ${ }^{32,33}$. In our simulations, this coupling is obtained by defining local effective temperatures for the acoustic and polar optical-phonon baths. However, in out-of-equilibrium conditions, the electron temperature is expected to deviate from that of phonons. To complete the physical picture of the system, we therefore also compute a non-ambiguous and thermodynamically-consistent values of electron temperature and electrochemical potential ${ }^{34,35}$. 
The paper is organized as follows. Section II describes the electronic quantum transport and heat transport models. In Section III we illustrate and discuss the simulation results in terms of current characteristics, coefficient of performance, phonon and electron temperatures, and variation of electrochemical potential. Finally, in Section IV we draw our concluding remarks.

\section{DESCRIPTION OF THE MODEL}

Figure (1.a) represents the band diagram and the working principle of the studied semiconductor heterostructure refrigerator (SHR). The cold electrons are injected into $4.8 \mathrm{~nm}$ wide GaAs quantum well (QW) by resonant tunneling through a $2.4 \mathrm{~nm}$ thick AlGaAs potential barrier. The cooling effect is driven by the transfer of energy from the lattice to electrons in the resonant state, mediated by the electron polar optical-phonon scattering. The thermally excited electrons are then removed from the well by thermionic emission over a $150 \mathrm{~nm}$ thick AlGaAs barrier, that serves as a thermal wall to reduce the heat backflow. The left and right GaAs access regions are $150 \mathrm{~nm}$ thick and n-doped with concentration $10^{18} \mathrm{~cm}^{-3}$.

\section{A. Electronic quantum transport model}

We use the NEGF transport equations expressed within the effective mass approximation to implement a one-dimensional (1D) quantum simulator along the heterostructure growth direction $(x)$. The single band effective mass Hamiltonian describes the $\Gamma$-valley of the conduction band of the III-V semiconductors. As the considered structure is translationally invariant in the perpendicular $y$ and $z$ directions, Born-Von-Karman periodic boundary conditions are applied on the transverse wave vector component $k_{t}$ such that $k_{t}=n_{k_{t}} \times 2 \pi / L_{t}$, with $L_{t}=50 \mathrm{~nm}$ and $n_{k_{t}}$ an integer indexing the transverse modes whose degeneracy is equal to $\pi\left(2 \times n_{k_{t}}+1\right)^{36}$. In the following, we briefly summarize the main features of the NEGF approach in matrix notation. We first define the retarded Green's function at the energy $E$ for each transverse mode $k_{t}$, 

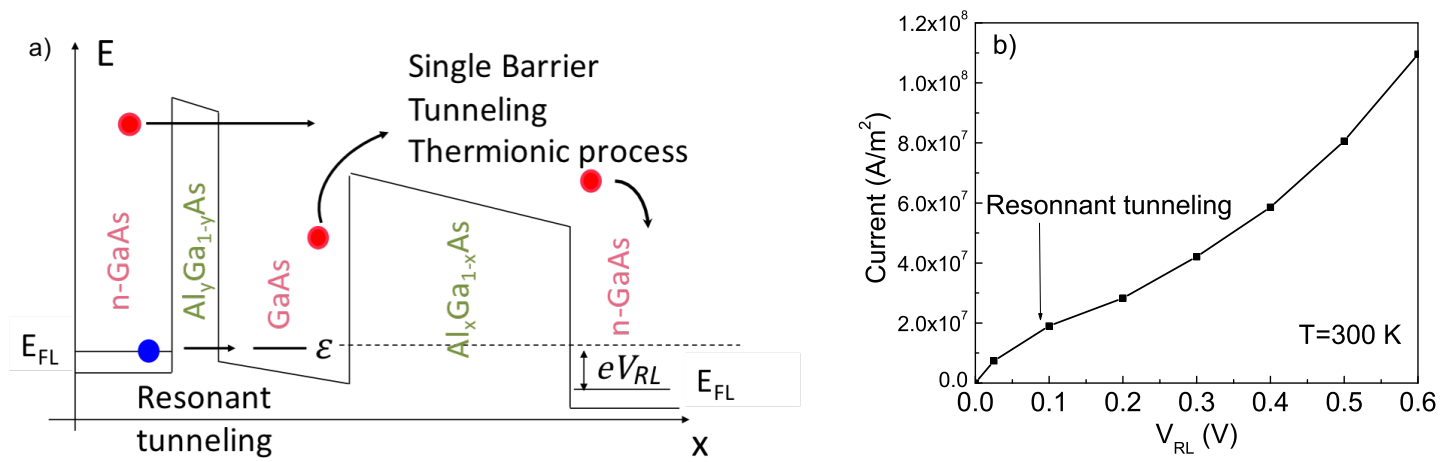

FIG. 1. a) Schematic band diagram of the studied semiconductor heterostructure refrigerator (SHR) device. The left and right $\mathrm{n}$-GaAs access regions are $150 \mathrm{~nm}$ wide and doped at a concentration of $10^{18} \mathrm{~cm}^{-3}$. The thin and thick AlGaAs barriers are $2.4 \mathrm{~nm}$ and $150 \mathrm{~nm}$ wide, respectively. The Al mole fractions are $y=0.3$ and $x=0.12$. The GaAs QW thickness is $4.8 \mathrm{~nm}$; b) Current density as a function of the voltage $V_{R L}$ applied between the left and right contacts, for a contact temperature $T=300 \mathrm{~K}$.

$$
G_{k_{t}}^{r}=\left[(E-V) I-H_{k_{t}}-\Sigma_{L, k_{t}}^{r}-\Sigma_{R, k_{t}}^{r}-\Sigma_{S, k_{t}}^{r}\right]^{-1}
$$

where $I$ is the identity matrix, $H_{k_{t}}$ represents the effective mass Hamiltonian for the transverse mode $k_{t}$ and $V$ is the electrostatic potential energy. $\Sigma_{L / R}^{r}$ and $\Sigma_{S}^{r}$ are the retarded self-energies for the left/right semi-infinite device contacts ${ }^{37}$ and scattering mechanisms, respectively.

From the retarded Green's function, the lesser/greater Green's functions are then obtained as

$$
G_{k_{t}}^{\lessgtr}=G_{k_{t}}^{r}\left(\Sigma_{L, k_{t}}^{\lessgtr}+\Sigma_{R, k_{t}}^{\lessgtr}+\Sigma_{S, k_{t}}^{\lessgtr}\right) G_{k_{t}}^{r \dagger},
$$

where the $\Sigma^{\lessgtr}$ are the lesser/greater self-energies, related to their retarded counterpart by

$$
\Sigma^{r}=\frac{1}{2}\left[\Sigma^{>}-\Sigma^{<}\right]
$$

Only acoustic- and polar optical-phonon interactions are considered, since non-polaroptical phonons turn out to be negligible in the materials constituting the device ${ }^{38}$. Interactions self-energies are calculated within the self-consistent Born approximation (SCBA) ${ }^{39-41}$. 
In our approach, the acoustic and polar optical-phonon baths locally follow a Bose-Einstein distribution, and are therefore assumed at equilibrium. On the other hand, acoustic and optical phonons can not be assumed at equilibrium with respect to each other, as the net anharmonic decay of optical phonons into acoustic, driven by an unbalance in their respective energy densities, plays a fundamental role in the thermal transport ${ }^{42}$. This is taken into account in our model by defining, at each position of the domain, two different temperatures, $T_{A C}$ and $T_{P O P}$ for the acoustic and polar optical-phonons, respectively. As detailed in the next subsection, these temperatures are self-consistently computed by coupling the electron transport equations with the heat equation. Within the elastic assumption, the SCBA self-energy for acoustic-phonons is independent of $k_{t}$ and can be expressed as ${ }^{39,43}$

$$
\Sigma_{A C}^{\lessgtr}(j, j, E)=\sum_{k_{t}^{\prime}} \pi\left(2 n_{k_{t}^{\prime}}+1\right) \frac{\Xi^{2} k_{\mathrm{B}} T_{A C}(j)}{\rho u_{s}^{2}} G_{k_{t}^{\prime}}^{\lessgtr}(j, j, E),
$$

where $\Xi$ is the deformation potential, $\rho$ is the mass density and $u_{s}$ is the sound velocity. In Eq.(4) index $j$ indicates the $x$ position along the discretized domain. Interactions with acoustic phonons are here assumed to be local and we then consider only the diagonal part of the Green's function. Such approximation has been demonstrated to be valid for acoustic phonons down to very low temperatures ${ }^{44}$. Concerning polar optical-phonons (POP), we adopt the diagonal expression of the scattering self-energy recently proposed as an effective description of their long range interactions ${ }^{45}$. For a given wave vector $k_{t}$, it can be shown that:

$$
\begin{aligned}
\Sigma_{P O P, k_{t}}^{\lessgtr}(j, j, E)= & \frac{\lambda M^{2}}{2 \pi S} \sum_{k_{t}^{\prime}}\left[\left(n_{L}(j)+1\right) G_{k_{t}^{\prime}}^{\lessgtr}\left(j, j, E \pm \hbar \omega_{L}\right)+\left(n_{L}(j)\right) G_{k_{t}^{\prime}}^{\lessgtr}\left(j, j, E \mp \hbar \omega_{q_{L}}\right)\right] \\
& \times \int_{\pi / L_{t}}^{\pi} \frac{\pi\left(2 n_{k_{t}^{\prime}}+1\right)}{\sqrt{\left(k_{t}-k_{t}^{\prime} \cos \theta\right)^{2}+\left(k_{t}^{\prime} \sin \theta\right)^{2}}} d \theta,
\end{aligned}
$$

with $n_{L}(j)=\left(e^{\left(\hbar \omega_{L}\right) /\left(k_{B} T_{P O P}(j)\right)}-1\right)^{-1}, M^{2}=2 \pi \hbar \omega_{L} e^{2}\left(\frac{1}{\epsilon_{\infty}}-\frac{1}{\epsilon_{0}}\right), \theta$ is the angle between $k_{t}$ and $k_{t}^{\prime}, S=\pi L_{t}^{2}$, and $\omega_{L}=35 \mathrm{meV} . M$ is the Fröhlich factor in which $\epsilon_{0}$ and $\epsilon_{\infty}$ represent the static and high frequency dielectric permittivity respectively. Finally $\lambda$ is a scaling factor which takes into account for the diagonal approximation. The value $\lambda=8$ used in this paper has been obtained within the comprehensive and physically-based analytical model proposed in Ref.45. 
The total phonon self-energy $\Sigma_{S, k_{t}}^{\lessgtr}$ for a given mode $k_{t}$ is then:

$$
\Sigma_{S, k_{t}}^{\lessgtr}=\Sigma_{A C}^{\lessgtr}+\Sigma_{P O P, k_{t}}^{\lessgtr} .
$$

Once the lesser/greater Green's function $G_{k_{t}}^{\lessgtr}$ of each mode $k_{t}$ is determined, physical quantities can be straightforwardly calculated ${ }^{39}$. The electron density $n$ along the transport direction reads:

$$
\begin{aligned}
n_{j} & =-2 \times \frac{i}{2 \pi} \sum_{k_{t}} \pi\left(2 n_{k_{t}}+1\right) \int_{-\infty}^{+\infty} G_{k_{t}}^{<}(j, j ; E) d E, \\
& =-i \int_{-\infty}^{+\infty} G^{<}(j, j ; E) d E,
\end{aligned}
$$

with $G^{<}(j, j ; E)=\sum_{k_{t}}\left(2 n_{k_{t}}+1\right) G_{k_{t}}^{<}(j, j ; E)$. The carrier current density (in $\left.\mathrm{A} / \mathrm{m}^{2}\right)$ flowing from position $j$ to $j+1$ along the $x$ direction is calculated from the off-diagonal elements $(j, j+1)$ of $G_{k_{t}}^{<}(i, j ; E)$ as

$$
\begin{aligned}
J_{j \rightarrow j+1} & =\int_{-\infty}^{+\infty} d E \frac{e}{\hbar} \sum_{k_{t}} \frac{\left(2 n_{k_{t}}+1\right)}{S}\left[H_{j, j+1} G_{k_{t}}^{<}(j+1, j ; E)-G_{k_{t}}^{<}(j, j+1 ; E) H_{j+1, j}\right] \\
& =\int_{-\infty}^{+\infty} \mathcal{J}_{j \rightarrow j+1}(E) d E .
\end{aligned}
$$

where $H_{j, j+1}$ corresponds to the nearest neighbors hopping terms in the discretized tightbinding like Hamiltonian and $\mathcal{J}_{j \rightarrow j+1}(E)$ is the current density spectrum (in $\mathrm{A} /\left(\mathrm{m}^{2} \cdot \mathrm{eV}\right)$ ). From Eq.(10) we can deduce the corresponding electronic energy current $^{46}$ :

$$
J_{j \rightarrow j+1}^{E}=\int_{-\infty}^{+\infty} E \mathcal{J}_{j \rightarrow j+1}(E) d E .
$$

In practice, the set of Eqs. (1)-(6) is solved self-consistently using a recursive algorithm ${ }^{37,47}$ until the criteria of convergence for both electron density and carrier current density are reached. The potential energy $V$ is self-consistently determined by nonlinearly coupling the transport equations (1)-(6) with the Poisson equation through the electron density.

\section{B. Heat transport model}

We compute the lattice temperature by solving the 1D heat equation along the $x$ direction. The discretized heat equation on the site $j$ reads:

$$
\left[-\frac{\partial}{\partial x}\left[\kappa_{t h}(x) \frac{\partial}{\partial x} T_{A C}(x)\right]\right]_{j}=Q_{j},
$$


where $\kappa_{t h}$ is the thermal conductivity. It is taken equal to the GaAs bulk value $(46 \mathrm{~W} /(\mathrm{m} \cdot \mathrm{K}))$ in all the device, except in the QW region, where it is set to $4 \mathrm{~W} /(\mathrm{m} \cdot \mathrm{K})$ in order to take into account the thermal resistance associated to the interface between different layers ${ }^{48,49}$. The temperature entering the heat equation is the one associated to the acoustic phonons, since they have a larger velocity than their polar optical counterparts and are mainly responsible of the heat transport ${ }^{50}$. Left and right reservoirs are assumed to be at the thermodynamic equilibrium at $T_{A C}=300 \mathrm{~K}$. This value is set by enforcing Dirichlet's boundary conditions at the left and right contact. This assumption corresponds to consider massive contacts with a sufficiently high thermal capacitance. $Q_{j}$ is the volumetric source term which corresponds to the heat power density (in $\mathrm{W} / \mathrm{m}^{3}$ ) generated by electron-phonon interactions. In the framework of the previously described electron transport formalism, it can be computed $\operatorname{as}^{33,46}$ :

$$
Q_{j}=-\nabla_{j} \cdot J^{E}
$$

A negative value of $Q_{j}$ corresponds to an energy transfer from the lattice to electrons, while a positive one describes the reverse phenomenon. In the absence of electron-phonon interactions, the charge conservation entails $\nabla \cdot \mathcal{J}(E)=0$ for each $E$, and, therefore, $Q=0$. From a physical point of view, electrons loose or increase their energy by scattering with polar optical-phonons. In turn, optical phonons decay into acoustic phonon modes, which sustain the thermal energy propagation along the device. In stationary conditions, the power transfer from optical to acoustic phonons must be equal to the heat power density $Q_{j}$ defined above. Within a relaxation time approximation ${ }^{42}$, we can thus write

$$
\frac{\left(T_{P O P}(j)-T_{A C}(j)\right) C_{P O P}}{\tau_{P O P \rightarrow A C}}=Q_{j},
$$

where $\tau_{P O P \rightarrow A C}$ is the relaxation time of polar optical-phonons into acoustic phonons $\left(\tau_{P O P \rightarrow A C}=4.1610^{-12} \mathrm{~s}\right)^{51}$ and $C_{P O P}$ is the thermal capacitance of the polar opticalphonons per unit volume $\left(C_{P O P}=1,72.10^{6} \mathrm{~J} /\left(\mathrm{m}^{3} \cdot \mathrm{K}\right)\right)^{51}$. The numerator of the left side expresses the average energy per unit volume exchanged between the polar optical and acoustic phonon baths in an interval $\tau_{P O P \rightarrow A C}$. Eq.(14) allows us to compute $T_{P O P}(j)$ from the knowledge of $Q_{j}$ and $T_{A C}(j)$. 
The computed values of $T_{A C}$ and $T_{P O P}$ are injected in Eq.(4) and Eq.(5), respectively. This establishes the coupling between the heat equation and the electron transport equations. The heat equation is iteratively solved together with the transport equations and the Poisson equation, until a global self-consistency is achieved.

\section{RESULTS}

\section{A. Local phonon temperature}

Figure (1.b) shows the electron current density as a function of the bias $V_{R L}$ between the right and left contacts, when the temperature in the reservoirs is set to $300 \mathrm{~K}$. The resonant tunneling can be easily identified. This physical phenomenon together with the thermionic emission above the thick barrier, are also visible in the current spectrum of Figure (2). Electrons are mainly injected through the thinner AlGaAs barrier at energies close to the resonant state in the GaAs QW. This filtering results in a charge accumulation in the well, which enhances the electron-phonon scattering rates ${ }^{39}$. On the other hand, no available states are present in the well at energies below the resonant state, and therefore electrons can only absorb phonons. If the energy difference between the resonant state and the top of the wider AlGaAs barrier is comparable with the energy of the polar optical-phonons (as it is the case in Fig.(2)), after absorbing a phonon, electrons can overcome the barrier and flow toward the right end of the device. As the potential of the wider AlGaAs barrier decreases, states at lower energies are made available for electrons. They then start thermalizing toward the Fermi-Dirac distribution by emitting polar optical-phonons and transferring heat power to the lattice. Figure (3.a) shows the corresponding electron energy current density (Eq.(11)) along the device. The current smoothly increases in the left access region, where phonon absorption dominates over emission ${ }^{33}$, increases more rapidly in the quantum well, where absorption is enhanced, and finally monotonically decreases as a consequence of electron thermalization.

The energy transferred between electrons and the lattice along the device can be exactly evaluated by the heat power density (Eq.(13)) as shown in Figure (3.b). The heat power density is negative on the left side, where a net transfer of energy from the lattice to electrons takes place, and positive on the right side. A negative peak of the heat power density, which 


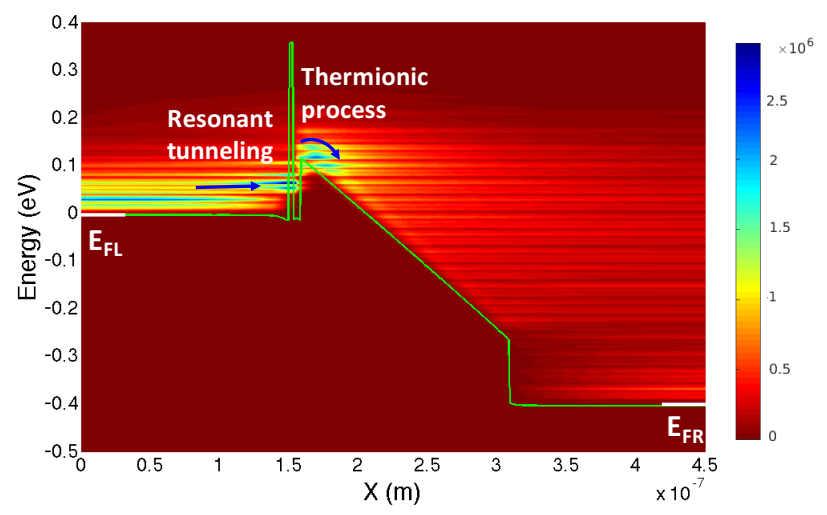

FIG. 2. Current density spectrum (arb. unit) obtained at $V_{R L}=0.4 \mathrm{~V}$ with a contact temperature $T=300 \mathrm{~K}$. Fermi levels of left and right contacts $\left(E_{F L}\right.$ and $E_{F R}$ respectively) are also represented. Arrows indicate the main current density flux in the QW resonant state and above the barrier, highlighting the physical processes involved. Potential profile is also plotted in solid line.
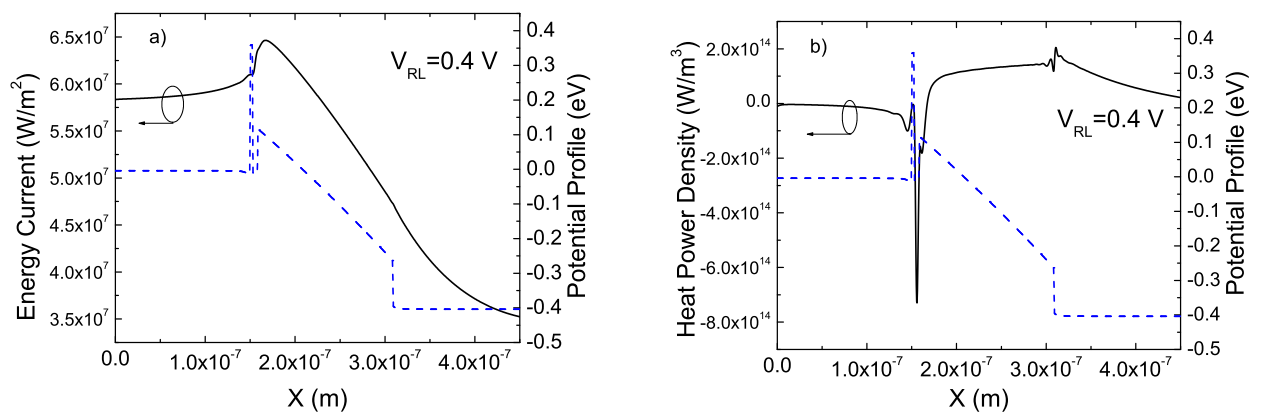

FIG. 3. a) Energy current density along the device (solid line) and b) heat power density along the device (solid line) at $V_{R L}=0.4 \mathrm{~V}$. The corresponding conduction band edge profile is plotted in both panels in dashed line.

accounts for most of the power extracted from the lattice, corresponds to the QW, where the absorption of polar optical-phonons mostly occurs.

Figure (4) shows the self-consistent polar optical-phonon temperature along the device for several values of $V_{R L}$. As expected, it decreases in the left region, while it increases on the right side. The temperature reaches its minimum in the QW. An increase of $V_{R L}$ entails an increase of the current (see Fig.(1-a)), and as a consequence, an increase of electron-phonon scattering. This results in an enhanced cooling effect. In the considered implementation, the maximum temperature difference that the refrigerator is able to establish is around $2 \mathrm{mK}$. 


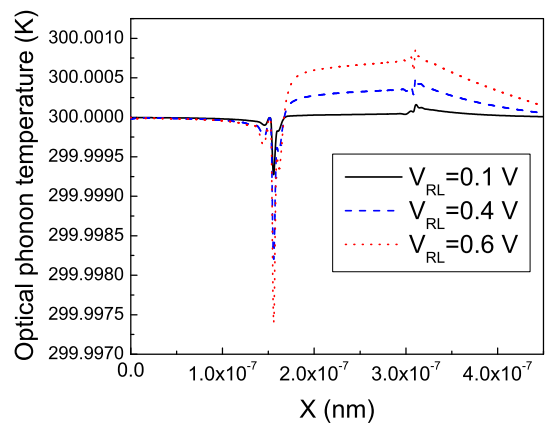

FIG. 4. Polar optical-phonon temperature profiles for different applied $V_{R L}$.

Such a small value is to be attributed to the high lattice thermal conductivity of AlGaAs and GaAs, which effectively level out the temperature inside the device.

Considering the present structure, the cooling power density of the device $J_{Q}$, obtained by integrating over $x$ the negative part of the heat power density (Eq.(13)), increases with the applied bias. It varies from $2,26 \cdot 10^{6} \mathrm{~W} / \mathrm{m}^{-2}$ at $V_{R L}=0.1 \mathrm{~V}$ to $7,5 \cdot 10^{6} \mathrm{~W} / \mathrm{m}^{-2}$ at $V_{R L}=0.6$ $\mathrm{V}$. Interestingly, the related coefficient of performance (COP), defined as the ratio of $J_{Q}$ by the applied power $\left.\left(P_{E l e c}=J \times V_{R L}\right)\right)$ follows the opposite trend. It goes from $11,7 \%$ at $V_{R L}=0.6 \mathrm{~V}$ up to $120 \%$ at $V_{R L}=0.1 \mathrm{~V}$. Such high COP values for low bias is physically sound as long as the integration of the heat power density over the whole device remains equal to $P_{\text {Elec }}$. It also confirms the real capacity of the considered device to cool the lattice.

\section{B. Local electron temperature}

As previously mentioned, temperature of phonons in a non-equilibrium system can significantly differ from its electronic counterpart. The comparison between the electron temperature and the one of optical phonons could provide information about the physics of the device and its possible optimizations. In this section, we calculate the local electronic temperature based on the virtual probe approach. Such a method can determine the local electronic temperature and electrochemical potential by cancelling the particle and energy currents between a floating probe and the SHR device. The probe is then in local thermodynamic equilibrium with the non-equilibrium structure. Stafford and co-workers ${ }^{34,35,52}$ recently showed that temperature and electrochemical potential determined within this approach are physically-consistent, as they are unique and fulfill the four laws of thermodynam- 
ics. More precisely, temperature and electrochemical potential are completely determined when the probe-system coupling is: i) maximally localized, to provide a good spatial resolution, ii) weak enough to generate a non-invasive measurement, iii) broadband, "to ensure that the measured physical properties depend on the energy spectrum of the structure and not on the one of the probe".

We then consider a thermoelectric probe at the position $j$ along the $x$-axis defined by the following self-energy (similar to the Büttiker probes ${ }^{53-55}$ ):

$$
\begin{aligned}
& \Sigma^{>}(j ; E)=-i\left[1-f_{F D}\left(E, \mu_{j}, T_{j}^{e}\right)\right] \operatorname{LDOS}(j ; E) \nu_{\text {coup }}, \\
& \Sigma^{<}(j ; E)=i f_{F D}\left(E, \mu_{j}, T_{j}^{e}\right) \operatorname{LDOS}(j ; E) \nu_{\text {coup }},
\end{aligned}
$$

where $f_{F D}$ is the Fermi-Dirac distribution of the electrons in the probe, $\mu_{j}$ and $T_{j}^{e}$ are respectively the local electrochemical potential and electronic temperature at the position $j$; $\operatorname{LDOS}(j ; E)=i \frac{\left[G^{>}(j, j ; E)-G^{<}(j, j ; E)\right]}{2 \pi}$ is the local density of states of the probe (taken equal to the one of the device) and $\nu_{\text {coup }}$ is the energy independent coupling strength between the probe and the system. In the considered case, the exact value of $\nu_{\text {coup }}$ is not important, as it will cancel out in the following computations.

By enforcing the simultaneous cancellation of the electron charge and energy currents between the device and the probe, we obtain a system of two coupled nonlinear equations in the unknowns $\mu_{j}$ and $T_{j}^{e}$ :

$$
\begin{aligned}
\Delta J(j) & =\int_{-\infty}^{+\infty} \Sigma^{>}(j ; E) G^{<}(j, j ; E) d E-\int_{-\infty}^{+\infty} G^{>}(j, j ; E) \Sigma^{<}(j ; E) d E=0 \\
\Delta J^{E}(j) & =\int_{-\infty}^{+\infty} E \Sigma^{>}(j ; E) G^{<}(j, j ; E) d E-\int_{-\infty}^{+\infty} E G^{>}(j, j ; E) \Sigma^{<}(j ; E) d E=0 .
\end{aligned}
$$

The system is iteratively solved at each position $j$ through a Newton-Raphson algorithm ${ }^{56}$. We remark that the model adopted for the probe fulfills the above-mentioned conditions ensuring the possibility to unambiguously define a local electron temperature and electrochemical potential. The system of Eqs. (17) and (18) is therefore expected to have a unique solution. These calculations are done as a post-processing step once the self-consistent lesser and greater Green's functions of the system are obtained.

Figure (5.a) shows the electron temperature along the device at $V_{R L}=0.1 \mathrm{~V}$. We first see that temperature varies over a much wider scale than in the polar optical-phonon case. The coldest temperature is still reached in the QW region. The temperature first drops by $\sim 22 \mathrm{~K}$ 

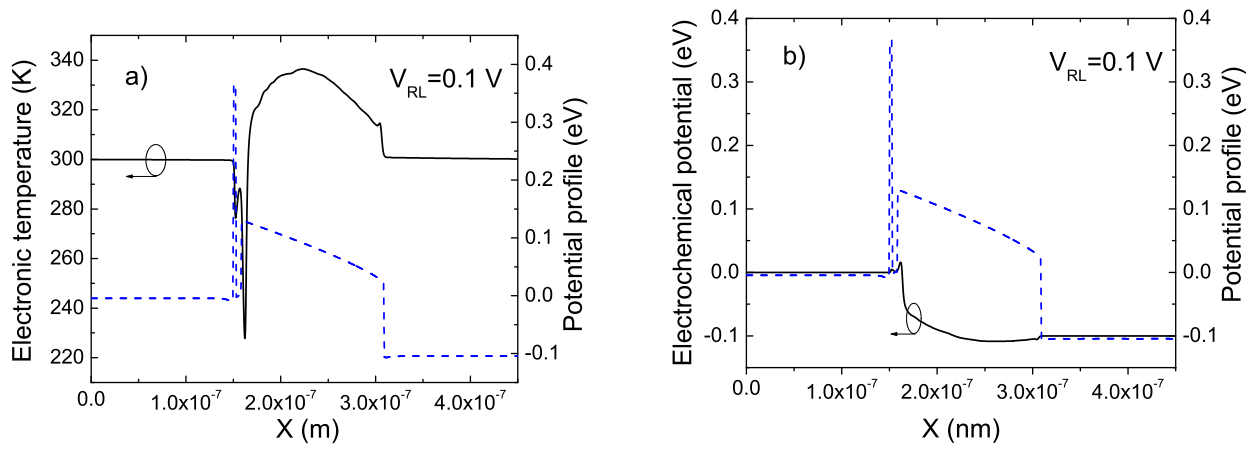

FIG. 5. a) Electronic temperature along the device obtained at $V_{R L}=0.1 \mathrm{~V}$ (solid line) and b) corresponding local electrochemical potential profile (solid line). In both panels the conduction band edge is plotted as reference in dashed line.

in the thin AlGaAs barrier, as a results of the energy filtering (which selects electrons close to the bottom of the conduction band). It then rises by $\sim 12 \mathrm{~K}$ in the quantum well, where electrons increase their kinetic energy due to a combined effect of polar optical phonons absorption and thermionic emission. At the onset of the thick AlGaAs barrier, the kinetic energy of electrons is mostly converted in potential energy, and the electron temperature drops again, until a minimum value of $236 \mathrm{~K}$. In the region between the quantum well and the right contact, the constant electric field associated to the linear bending of the AlGaAs barrier induces an increase of the average electron kinetic energy. This results in an increase of the electron temperature up to $337 \mathrm{~K}$. By moving further toward the right contact, electrons start thermalizing and the temperature decreases. Electrons participating in the transport correspond to a much smaller charge density with respect to the total mobile charge in the doped access regions $\left(10^{15}\right.$ against $\left.10^{18} \mathrm{~cm}^{-3}\right)$. Therefore, the hot electrons reaching the right access region are screened along few nanometers and the global electronic temperature recovers its contact equilibrium value of $300 \mathrm{~K}$.

For the sake of completeness, the corresponding plot of the local electrochemical potential is shown in Figure (5.b). Electrochemical potential remains typically between the two Fermi levels of the contacts (i.e. between $0 \mathrm{eV}$ and $-0.1 \mathrm{eV}$ ). It slightly increases in the $\mathrm{QW}$ since the average energy of the electrons tends to also increase. Conversely, the electrochemical potential decreases in the thick AlGaAs barrier since the emission of polar optical-phonons 

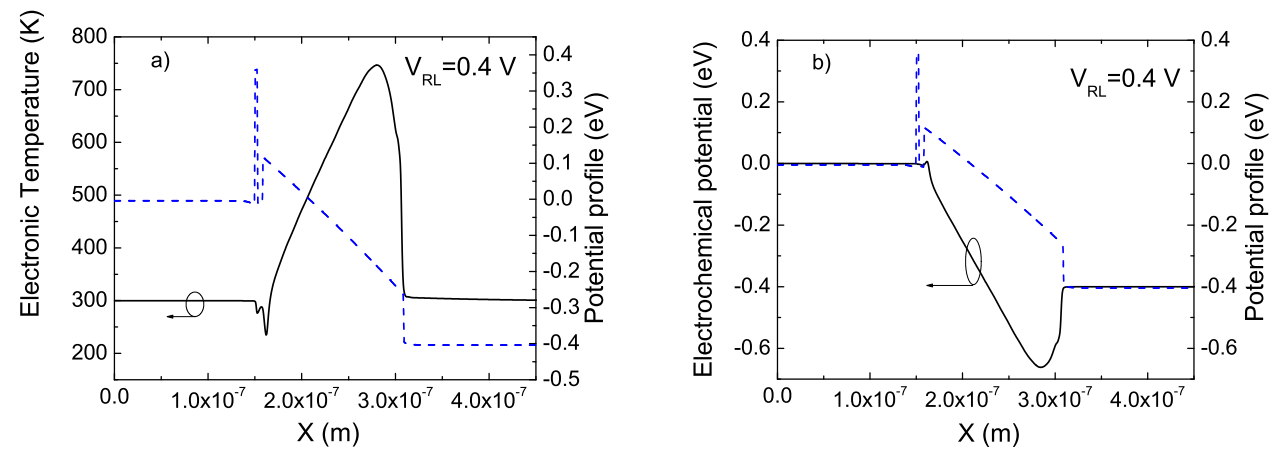

FIG. 6. a) Electronic temperature along the device obtained at $V_{R L}=0.4 \mathrm{~V}$ (solid line) and b) corresponding local electrochemical potential profile (solid line). In both panels the conduction band edge is plotted as reference in dashed line.

leads to a decrease of the average electron energy.

Figures (6.a) and (6.b) show the electron temperature and the corresponding electrochemical potential obtained at $V_{R L}=0.4 \mathrm{~V}$. According to the discussion in Subsection (III A), the higher electron current density enhances the phonon absorption rate, leading to a higher electronic temperature in the quantum well. The minimum value of the temperature at the onset of the thick AlGaAs barrier consequently decreases to $207 \mathrm{~K}$. On the other hand, the maximum temperature increases to $740 \mathrm{~K}$. This is is consistent with the four times larger electric field in the region of the thick AlGaAs barrier (cf. the steeper slope of the potential profile in Figure (6) with respect to Figure (5)), which drives hot electrons appreciably more out-of-equilibrium. The capability of the SHR to modulate over such wide ranges the electronic temperature is in agreement with the high values of cooling power densities attainable and confirms the potentialities of the device.

In order to validate this approach of measurement of the electronic temperature and electrochemical potential in non-equilibrium regime, we consider the following electron density expression:

$$
n_{j}^{e q}=\int_{-\infty}^{+\infty} \operatorname{LDOS}(j ; E) f_{F D}\left(E, \mu_{j}, T_{j}^{e}\right) d E
$$

A consistency check can rely on the comparison between the charge density values obtained from Eq.(19) and those obtained by solving the NEGF transport equations (Eq.(8)). In Figure (7) such a comparison is proposed for $V_{R L}=0.4 \mathrm{~V}$. The excellent agreement between 


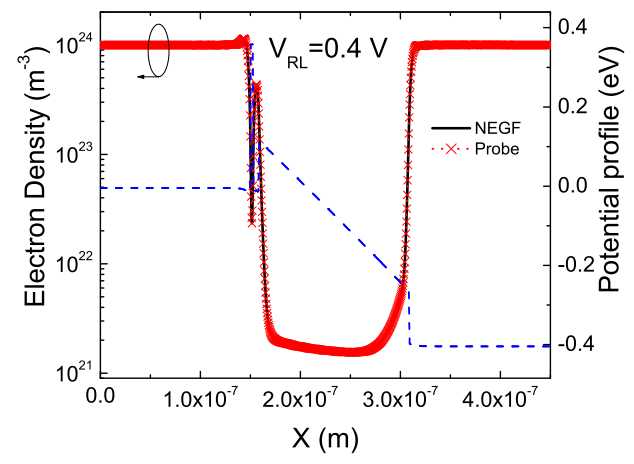

FIG. 7. Electron density along the device at $V_{R L}=0.4 \mathrm{~V}$, obtained by solving the NEGF transport equations (solid line), and from Eq.(19) (crosses). The conduction band edge is plotted for reference in dashed line.

the two computational approaches definitely supports the values of $T^{e}$ and $\mu$.

\section{CONCLUSION}

We numerically investigated the cooling properties of a GaAs/AlGaAs SHR based on a combination of resonant-tunneling and thermionic processes. By adopting a full-quantum approach, we have resolved the electric and thermal effects at play and described their reciprocal influence. The physical picture provided has been further complemented by introducing, through a virtual probe approach, a thermal characterization of electrons. In the considered implementation of the device, we have found power cooling densities of the order of $10^{2} \mathrm{~W} / \mathrm{cm}^{2}$ and a maximum COP of $120 \%$ at low bias $\left(V_{R L}=0.1 \mathrm{~V}\right)$. The difference of lattice temperature the device is able to maintain is, however, limited to few $\mathrm{mK}$, due to the high thermal conductivity of GaAs and AlGaAs. Unlike the phonon case, electronic temperature can vary up to several hundred of kelvins. At high applied bias (i.e. $V_{R L}>0.4 \mathrm{~V}$ ) the electronic temperature can be reduced by $90 \mathrm{~K}$ in the QW region, while exceeding 700 $\mathrm{K}$ in the hot region. Besides the obvious advantage that could derive by considering materials with lower thermal conductivities, a viable option to improve the performance of the refrigerator consists in increasing the doping of the access regions. For a given supply power, this is expected to increase the electron density in the quantum well, and therefore the rate of phonon absorption. An optimization of the process of heat extraction from the lattice 
could be also performed by finely tuning the energy of the resonant level. A higher thermal isolation between the hot and cold side of the device could be obtained by tailoring the shape of the wider AlGaAs barrier, particularly by flattening its top in order to ballistically guide electrons to thermalize farer from the quantum well. Finally, looking at a suitable trade-off between a decrease of the thermal conductivity by including superlattice sections and the concurrent degradation of electron mobility can be also envisaged.

\section{ACKNOWLEDGEMENT}

This work was granted access to the HPC resources of Aix-Marseille Universite financed by the project Equip@Meso (ANR-10-EQPX-29-01).

* bescond@iis.u-tokyo.ac.jp

1 R. Rhyner and M. Luisier, Nano Lett. 16, 1022 (2016).

2 K. L. Grosse, M. H. Bae, F. Lian, E. Pop, and W. P. King, Nat. Nanotechnol. 6, 287 (2011).

3 J. Srinivasan, S. V. Adve, P. Bose, and J. A. Rivers, in Dependable Systems and Networks, 2004 International Conference on (IEEE, 2004) pp. 177-186.

4 X. S. Sun, T. J. Silverman, Z. G. Zhou, M. R. Khan, P. Bermel, and M. A. Alam, IEEE-J. Photovolt. 7, 566 (2017).

5 J. Muhonen, M. Meschke, and J. Pekola, Nano Lett. 12, 758 (2012).

6 A. Shakouri, Annu. Rev. Mater. Res. 41, 399 (2011).

7 F. Giazotto, T. T. Heikkila, A. Luukanen, A. M. Savin, and J. P. Pekola, Rev. Mod. Phys. 78, $217(2006)$.

8 L. Hicks and M. S. Dresselhaus, Physical review B 47, 16631 (1993).

9 R. Venkatasubramanian, E. Siivola, T. Colpitts, and B. O'quinn, Nature 413, 597 (2001).

10 T. Harman, P. Taylor, M. Walsh, and B. LaForge, Science 297, 2229 (2002).

11 A. I. Hochbaum, R. Chen, R. D. Delgado, W. Liang, E. C. Garnett, M. Najarian, A. Majumdar, and P. Yang, Nature 451, 163 (2008).

12 A. I. Boukai, Y. Bunimovich, J. Tahir-Kheli, J.-K. Yu, W. A. Goddard Iii, and J. R. Heath, Nature 451, 168 (2008). 
13 Y. Lu, L. Zeng, A. J. Minnich, M. S. Dresselhaus, and G. Chen, Nature Nanotech. 10, 701 (2015).

14 J. Mairen, R. Anufriev, A. Ramiere, R. Yanagisawa, S. Volz, and M. Nomura, Sci. Adv. 3, e1700027 (2017).

15 R. Anufriev and M. Nomura, Phys. Rev. B 95, 155432 (2017).

16 M. D. Ulrich, P. A. Barnes, and C. B. Vining, J. Appl. Phys. 90, 1625 (2001).

17 G. D. Mahan, J. Appl. Phys. 76, 4362 (1994).

18 G. D. Mahan, J. O. Sofo, and M. Bartkowiak, J. Appl. Phys. 83, 4683 (1998).

19 G. D. Mahan and L. M. Woods, Phys. Rev. Lett. 80, 4016 (1998).

20 M. D. Ulrich, P. A. Barnes, and C. B. Vining, J. Appl. Phys. 92, 245 (2002).

21 R. Kim, C. Jeong, and M. S. Lundstrom, J. Appl. Phys. 107, 054502 (2010).

22 S. Wang and N. Mingo, Phys. Rev. B 79, 115316 (2009).

23 T. Humphrey and H. Linke, Phys. Rev. Lett. 94, 096601 (2005).

24 T. Humphrey, M. O’Dwyer, and H. Linke, J. Phys. D: Appl. Phys. 38, 2051 (2005).

25 R. S. Whitney, Phys. Rev. Lett. 112, 130601 (2014).

26 R. S. Whitney, Phys. Rev. B 91, 115425 (2015).

27 T. E. Humphrey, R. Newbury, R. P. Taylor, and H. Linke, Phys. Rev. Lett. 89, 116801 (2002).

28 A. Crépieux and F. Michelini, J. Phys.: Condens. Matter 27, 015302 (2015).

29 J. Azema, P. Lombardo, and A.-M. Daré, Phys. Rev. B 90, 205437 (2014).

30 Y. Zhang, J. Christofferson, A. Shakouri, G. Zeng, J. E. Bowers, and E. T. Croke, IEEE Transactions on components and packaging Technologies 29, 395 (2006).

31 K. A. Chao, M. Larsson, and A. G. Malshukov, Appl. Phys. Lett. 87, 022103 (2005).

32 R. Kim and M. Lundstrom, J. Appl. Phys. 110, 034511 (2011).

33 M. Pala and A. Cresti, J. Appl. Phys. 117, 084313 (2015).

34 C. A. Stafford, Phys. Rev. B 93, 245403 (2016).

35 A. Shastry and C. A. Stafford, Phys. Rev. B 94, 155433 (2016).

36 N. Cavassilas, F. Michelini, and M. Bescond, J. of Renewable and Sustainable Energy 6, 011203 (2014).

37 D. K. Ferry and S. M. Goodnick, Transport in Nanostructures (Cambridge University Press, Cambridge, U.K., 1997).

38 A. Price and A. Martinez, J. Appl. Phys. 12, 6670 (2015). 
39 S. Jin, Y. J. Park, and H. S. Min, J. Appl. phys. 99, 123719 (2006).

40 Y. Lee, M. Lannoo, N. Cavassilas, M. Luisier, and M. Bescond, Phys. Rev. B 93, 205411 (2016).

41 A. Svizhenko and M. P. Anantram, IEEE-Trans. Electron Dev. 50, 1459 (2003).

42 J. Lai and A. Majumdar, Journal of Applied Physics 79, 7353 (1996).

43 C. Jacoboni and L. Reggiani, Rev. Mod. Phys. 55, 645 (1983).

44 M. Bescond, H. Carrillo-Nuez, S. Berrada, N. Cavassilas, and M. Lannoo, Solid State Electron. 122, 1 (2016).

45 M. Moussavou, M. Bescond, N. Cavassilas, and M. Lannoo, To be submitted (2017).

46 R. Lake and S. Datta, Phys. Rev. B 117, 164501 (1992).

47 M. Lopez-Sancho, J. Lopez-Sancho, and J. Rubio, J. Phys. F: Met. 14, 1205 (1984).

48 A. Sood, J. A. Rowlette, C. G. Caneau, E. Bozorg-Grayeli, M. Asheghi, and K. E. Goodson, Appl. Phys. Lett. 105, 051909 (2014).

49 M. N. Luckyanova, J. A. Johnson, A. A. Maznev, J. Garg, A. Jandl, M. T. Bulsara, E. A. Fitzgerald, K. A. Nelson, and G. Chen, Nano Lett. 13, 3973 (2013).

50 E. Pop, S. Sinha, and K. E. Goodson, in SISPAD Tech. Dig. (IEEE, 2003) pp. 121-124.

51 T. Luo, J. Garg, J. Shiomi, K. Esfarjani, and G. Chen, EPL 101, 16001 (2013).

52 J. Meair, J. P. Bergfield, C. A. Stafford, , and P. Jacquod, Phys. Rev. B 90, 035407 (2014).

53 M. Büttiker, Phys. Rev. B 33, 3020 (1986).

54 G. Romano, A. Gagliardi, A. Pecchia, and A. D. Carlo, Phys. Rev. B 81, 115438 (2010).

55 R. Rhyner and M. Luisier, Phys. Rev. B 89, 235311 (2014).

56 R. Venugopal, M. Paulsson, S. Goasguen, S. Datta, and M. S. Lundstrom, J. Appl. Phys. 93, $5613(2003)$.

57 G. Klimeck, R. Lake, C. L. Fernando, R. C. Bowen, D. Blanks, M. Leng, T. Moise, Y. Kao, and W. R. Frensley, in Quantum Devices and Circuits (Imperial Press, London, 1996) pp. 154-159. 